Portland State University

PDXScholar

Electrical and Computer Engineering Faculty

Publications and Presentations

$6-20-1993$

\title{
Scattering of a Light Beam from Waves at an Air-Sea Interface
}

Donald D. Duncan

Portland State University

W. H. Carter

Follow this and additional works at: https://pdxscholar.library.pdx.edu/ece_fac

Part of the Electrical and Computer Engineering Commons

Let us know how access to this document benefits you.

\section{Citation Details}

W. H. Carter and D. D. Duncan, "Scattering of a Light Beam from Waves at an Air-Sea Interface," Applied Optics, V. 32, pp. 3286-3294 (1993)

This Article is brought to you for free and open access. It has been accepted for inclusion in Electrical and Computer Engineering Faculty Publications and Presentations by an authorized administrator of PDXScholar. Please contact us if we can make this document more accessible: pdxscholar@pdx.edu. 


\title{
Scattering of a light beam from waves at an air-sea interface
}

\author{
William H. Carter and Donald D. Duncan
}

\begin{abstract}
The authors report a new theory that describes the scattering of an upward propagating laser beam from the sea through the air-sea interface in the presence of sea waves. The sea is assumed to be a uniform dielectric and conventional scattering theory is employed by using a modification to the first Born approximation that permits treatment of surface refraction phenomena. Methods of statistical radiometry are also used in a new manner by assuming that the surface scattering function for the sea waves can be treated by a quasi-homogeneous source model to calculate the second-order correlation functions for the partially coherent scattered field. These correlation functions yield a simple expression for the radiant intensity of the scattered field as the convolution of the Fourier transform of the complex degree of spectral coherence for the sea waves with the squared modulus of the angular spectrum of plane waves for the incident laser beam. We believe that this theory is a significant improvement over the models that are usually used for modeling this phenomenon.
\end{abstract}

\section{Introduction}

We report a theoretical study of the scattering of an initially coherent laser beam that propagates from the sea into the air at the air-sea interface in the presence of sea waves. For this study we used a scattering theory model ${ }^{1}$ based on an improved first Born approximation described in this paper and methods developed in statistical radiometry ${ }^{2}$ by using the quasi-homogeneous source model. ${ }^{3}$ We obtained an expression for the radiant intensity distribution in the far field of the radiation that was scattered from the illuminated area on the sea surface. This problem is important to the U.S. Navy for developing laser systems to communicate from submarines to aircraft.

In previous engineering studies ${ }^{4}$ it is usually assumed that the radiation is thermal so that geometric optical techniques (which ignore interference effects) can be used to calculate the transmission of the light intensity through the air-sea interface. The air-sea surface is usually treated by tracing rays through multiple reflections by using Monte Carlo computer simulations. ${ }^{5}$ And the structure of the surface itself is usually modeled deterministically by using mea-

W. H. Carter is with the U.S. Naval Research Laboratory, Washington, D.C. 20375-5351. D. D. Duncan is with the Applied Physics Laboratory, The Johns Hopkins University, Johns Hopkins Road, Laurel, Maryland 20723-6099.

Received 15 January 1992.

0003-6935/93/183286-09\$06.00/0.

( 1993 Optical Society of America. sured sea wave data.6,7 None of these techniques accounts properly for the high coherence of incident light, if a laser beam is being considered, or for the effects of the motion of the sea waves on reducing the coherence of the transmitted light. The coherence of the transmitted light frequently has a large effect on the radiant intensity that is measured far above the surface. ${ }^{3}$ Therefore it is important to take into account the coherence properties of the transmitted light that are due to the effects of the sea waves.

In the present analysis we make no assumptions about the state of coherence of the light at the sea surface. Instead we assume that the sea waves themselves have an area of coherence that is spatially stationary in a statistical sense and is also small relative to the distance over which the average scattering potential of the interface changes appreciably. From these assumptions about the interface we calculated the second-order correlation functions that describe the state of partial coherence for the scattered light.

It is believed that this analysis is a step toward a more realistic model for this important engineering problem.

We also study the scattering of a single plane wave that is incident on a deterministic air-sea surface from below by using this model. We find that the improved first Born approximation (which requires an assumption of homogeneity for the scattering potential of the sea) leads to an expression that is similar to Snell's law. A similar theory that uses 
only the conventional first Born approximation does not treat refraction at the surface. Thus the improved first Born approximation used here is significantly better for treating surface scatter in applications in which the scattering medium is homogeneous.

In Section 2 we derive equations for the angular spectrum of the scattered field as a function of the surface scattering function for sea waves. In Section 3 we use these equations to study the scattering of a single, normally incident, plane wave by a sea surface that is characterized by a deterministic sea wave function. In Section 4 we use the equations from Section 2 to calculate the radiant intensity of the scattered field from a sea wave structure that is random and that is assumed to be represented by a quasi-homogeneous source model. Finally in Section 5 we generalize the theory developed in Section 4 to treat the scattering of an arbitrary, coherent laser beam from this sea surface.

\section{Surface Scattering Theory}

Consider a monochromatic, coherent laser beam that propagates from sources within the sea and is incident from below on the surface as shown in Fig. 1 .

The incident laser beam is partly reflected at the sea surface and partly transmitted out into the air as shown in the figure. We are concerned with the effects of sea waves on the radiation pattern of the transmitted light in the far field of the surface. We can neglect the reflected part of the laser beam. In order to apply conventional scattering theory to the problem of the interaction of the laser beam with the sea waves, it is convenient to treat the sea as an infinite dielectric slab, with constant index of refraction $n_{0}$ immersed in free space. Let the $x-y$ plane be just below the surface of the sea and let the sea extend down to some constant finite depth $d$, as shown in Fig. 2. Later in this analysis we let $d$ approach infinity so that the sea fills the $z<0$ half-space.

For purposes of our model we assume that the laser beam originates in free space and propagates from the left, through a fictional planar interface, into the slab that represents the water. We can neglect reflection and refraction at this first surface since we are not interested in the fields in the fictional region to the left of the slab. The total light field inside the water (neglecting the reflection of the laser beam from the

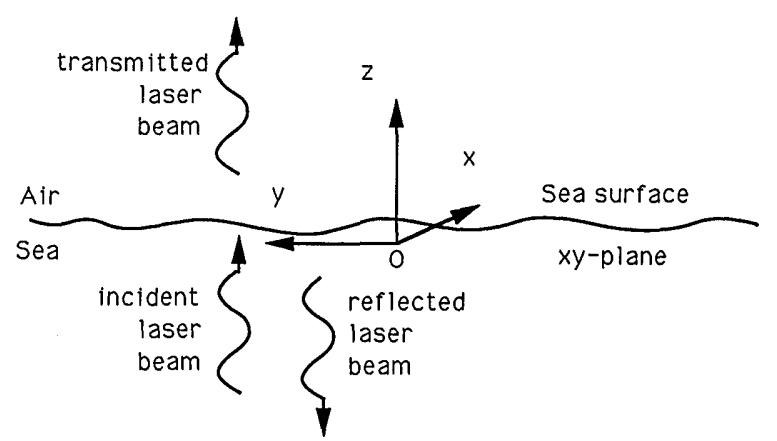

Fig. 1. Actual orientation of the sea surface and the coordinate system used in this analysis.

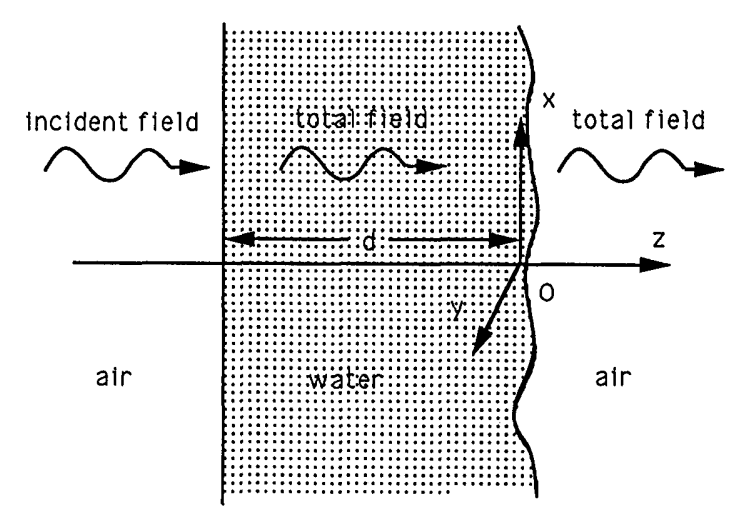

Fig. 2. Dielectric slab used to treat sea wave scattering of coherent light.

sea surface to the right) can be decomposed into the incident field plus a scattered field. To the right of the slab the total field, which corresponds to the laser beam that is transmitted through the sea surface into the air, is also decomposed into an incident field plus a scattered field. It is the radiant intensity of the scattered part of this transmitted laser beam that we study.

Following the usual procedure for treating scattering from objects contained within an infinite slab, ${ }^{1}$ we note that the total field amplitude at any point in the source-free region of space shown in Fig. 2 is given by the homogeneous Helmholtz equation ${ }^{8}$ :

$$
\left[\nabla^{2}+n^{2}(\mathbf{x}) k^{2}\right] \psi_{t}(\mathbf{x})=0,
$$

where $\mathbf{x}$ is the radius vector from the origin to some arbitrary point, $n(\mathbf{x})$ is the index of refraction at the point, and $k=2 \pi / \lambda$ is the propagation constant for free space. The incident field is defined to have a field amplitude given by

$$
\left(\nabla^{2}+k^{2}\right) \psi_{i}(\mathbf{x})=0
$$

so that it is the field that would be present everywhere if the dielectric slab in Fig. 2 were absent. The scattered field is defined to be the difference between the incident field and the total field, i.e.,

$$
\psi_{s}(\mathbf{x})=\psi_{t}(\mathbf{x})-\psi_{i}(\mathbf{x})
$$

The scattered field represents the perturbation to the incident field that is due to the presence of the slab. By substitution of Eq. (3) into Eq. (1) and using Eq. (2), we have

$$
\left(\nabla^{2}+k^{2}\right) \psi_{s}(\mathbf{x})=k^{2}\left[1-n^{2}(\mathbf{x})\right] \psi_{t}(\mathbf{x})
$$

for the scattered field.

By solving Eq. (4) for the scattered field amplitude, we get $^{8}$

$$
\psi_{s}(\mathbf{x})=\iiint_{\text {sea slab }} F\left(\mathbf{x}^{\prime}\right) \psi_{t}\left(\mathbf{x}^{\prime}\right) \frac{\exp \left(i k\left|\mathbf{x}-\mathbf{x}^{\prime}\right|\right)}{\left|\mathbf{x}-\mathbf{x}^{\prime}\right|} \mathrm{d}^{3} \mathbf{x}^{\prime},
$$


where the scattering potential is defined, as usual, by

$$
F\left(\mathbf{x}^{\prime}\right)=\frac{-k^{2}}{4 \pi}\left[1-n^{2}\left(\mathbf{x}^{\prime}\right)\right],
$$

and the domain of integration in Eq. (5) is over all the points that are within the water. If there were no sea waves this would be just the slab shown in Fig. 2. However, if sea waves are present, the integration must extent above the $z=0$ plane to some plane $z=$ $z_{+}$that is above the tops of the waves.

We note that, since the total field includes the scattered field, Eq. (5) is not a solution for the scattered field. To find a useful expression for the scattered field it is usually necessary to make a limiting approximation. The most common, the first Born approximation, assumes that the total field inside the slab can be accurately approximated by the incident field alone. Substituting the incident field for the total field in Eq. (5) gives an approximate solution for the scattered field. The first Born approximation is valid only if the scatterer is weak so that the scattered field makes only a small contribution to the total field inside the slab. This is clearly not true of seawater. There is a much better approximation that we can make if the index of refraction inside the water is constant. By using macroscopic electromagnetic theory, we assume that the main effect of the seawater is to modify the incident field by changing the propagation constant wherever it appears in the expression for the incident field from that for free space to that within the slab, i.e., $k \rightarrow n_{0} k$. Thus we replace the total field inside Eq. (5) with a modified incident field $\bar{\psi}_{i}(\mathbf{x})$ to obtain

$$
\psi_{s}(\mathbf{x})=\iiint_{\text {sea slab }} F\left(\mathbf{x}^{\prime}\right) \bar{\psi}_{i}\left(\mathbf{x}^{\prime}\right) \frac{\exp \left(i k\left|\mathbf{x}-\mathbf{x}^{\prime}\right|\right)}{\left|\mathbf{x}-\mathbf{x}^{\prime}\right|} \mathrm{d}^{3} \mathbf{x}^{\prime} .
$$

Since we are interested only in the interaction of the laser beam with the sea waves and the effects this has on the transmitted light, we can restrict our calculation to the scattered field at points in the $z>0$ half-space. This corresponds to the region to the right of the slab in Fig. 2. Over this region, we can transform Eq. (7) by substitution from Weyl's integral [see Ref. 9 and Ref. 10, Eq. (55)],

$$
\begin{aligned}
\frac{\exp \left(i k\left|\mathbf{x}-\mathbf{x}^{\prime}\right|\right)}{\left|\mathbf{x}-\mathbf{x}^{\prime}\right|}= & \frac{-i k}{2 \pi} \int_{-\infty}^{\infty} \int_{-\infty}^{\infty} \frac{1}{m} \exp \left\{i k \left[p\left(x-x^{\prime}\right)\right.\right. \\
& \left.\left.+q\left(y-y^{\prime}\right)+m\left|z-z^{\prime}\right|\right]\right] \mathrm{d} p \mathrm{~d} q,
\end{aligned}
$$

into Eq. (7) to yield an expansion of the scattered field into an angular spectrum of plane waves in the form

$$
\begin{aligned}
\psi_{s}(\mathbf{x}) & =\int_{-\infty}^{\infty} \int_{-\infty}^{\infty} A(p, q) \exp [i k(p x+q y+m z)] \mathrm{d} p \mathrm{~d} q \\
m & =\left(1-p^{2}-q^{2}\right)^{1 / 2} \quad \text { if } p^{2}+q^{2} \leq 1 \\
& =i\left(p^{2}+q^{2}-1\right)^{1 / 2} \text { otherwise, }
\end{aligned}
$$

where the plane-wave amplitudes are given by

$$
\begin{aligned}
A(p, q) & =\frac{1}{\lambda^{2} m} \int_{-\infty}^{\infty} \int_{-\infty}^{\infty} G\left(x^{\prime}, y^{\prime}\right) \exp \left[-i k\left(p x^{\prime}+q y^{\prime}\right)\right] \mathrm{d} x^{\prime} \mathrm{d} y^{\prime} \\
& =\frac{\tilde{G}(p, q)}{m}
\end{aligned}
$$

In deriving Eqs. (9) and (10) we have defined

$$
\tilde{G}(p, q)=\frac{1}{\lambda^{2}} \int_{-\infty}^{\infty} \int_{-\infty}^{\infty} G\left(x^{\prime}, y^{\prime}\right) \exp \left[-i k\left(p x^{\prime}+q y^{\prime}\right)\right] \mathrm{d} x^{\prime} \mathrm{d} y^{\prime},
$$

the Fourier transform of the surface scattering function, which is defined by

$$
G\left(x^{\prime}, y^{\prime}\right)=-i \lambda \int_{-\mathrm{d}}^{z_{+}} F\left(\mathbf{x}^{\prime}\right) \bar{\psi}_{i}\left(\mathbf{x}^{\prime}\right) \exp \left(-i k m z^{\prime}\right) \mathrm{d} z^{\prime},
$$

where $z=z_{+}$defines the plane just above the sea surface and above the tops of any sea waves that might be present.

We can continue our analysis in several different ways from this point. We can either assume some deterministic model for the sea waves or use a statistical model instead. Also, we can assume that the incident laser beam can be treated as a simple plane wave to make the analysis simple, or we can use a more realistic model and obtain more complicated results. Each of these approaches has its advantages. In Section 3 we use the simplest model of a single incident plane wave that propagates parallel to the $z$ axis and is scattered by waves of known form.

\section{Scattering of a Normally Incident Plane Wave From a Deterministic Sea Wave Structure}

Let the laser beam be approximated by a single plane wave incident parallel to the $z$ axis. As discussed above, we assume that the total field inside the sea can be usefully approximated by the modified incident field given by

$$
\psi_{t}\left(\mathbf{x}^{\prime}\right) \cong \bar{\psi}_{i}\left(\mathbf{x}^{\prime}\right)=\psi_{0} \exp \left(i k n_{0} z\right) .
$$

In this calculation we assume that the sea waves can be characterized by a known, deterministic waveform $f(x, y)$, which is defined such that the surface of the sea embedded in three-dimensional space is given by $f(x, y)=z$. Since the index of refraction is given by $n_{0}$ everywhere within the water and unity everywhere else, from Eq. (6) we have the scattering potential:

$$
\begin{aligned}
F\left(\mathbf{x}^{\prime}\right) & =\frac{-k^{2}}{4 \pi}\left[1-n_{0}^{2}\right] \triangleq F_{0} \text { if }-\mathrm{d} \leq z^{\prime} \leq f\left(x^{\prime}, y^{\prime}\right) \\
& =0 \text { otherwise. }
\end{aligned}
$$

After substitution from relation (13) and Eq. (14) into Eq. (12), the expression for the surface scattering 
function becomes

$$
G\left(x^{\prime}, y^{\prime}\right)=-i \lambda \psi_{0} F_{0} \int_{-\mathrm{d}}^{f\left(x^{\prime}, y^{\prime}\right)} \exp \left[-i k\left(m-n_{0}\right) z^{\prime}\right] \mathrm{d} z^{\prime} .
$$

The integral in Eq. (15) can be evaluated by use of the Fourier transform of the Heaviside function as tabulated by Bracewell (see Ref. 11, p. 420), i.e.,

$$
\int_{0}^{\infty} \exp (-2 \pi i x \xi) \mathrm{d} \xi=\frac{\delta(x)}{2}-\frac{i}{2 \pi x} .
$$

To evaluate this integral we let $d$ become large and employ Eq. (16) to obtain

$$
\begin{aligned}
G\left(x^{\prime}, y^{\prime}\right) \underset{\mathrm{d} \rightarrow \infty}{\approx} & \frac{-i \lambda^{2} \Psi_{0} F_{0}}{2}\left\{\delta\left(m-n_{0}\right)+\frac{i}{\pi\left(m-n_{0}\right)}\right. \\
& \left.\times \exp \left[-i k\left(m-n_{0}\right) f\left(x^{\prime}, y^{\prime}\right)\right]\right\} .
\end{aligned}
$$

On substitution from approximation (17) into Eq. (10), we have

$$
\begin{aligned}
A(p, q)= & \frac{-i \lambda^{2} \Psi_{0} F_{0}}{2 m \lambda^{2}} \\
& \times \int_{-\infty}^{\infty} \int_{-\infty}^{\infty}\left\{\delta\left(m-n_{0}\right)+\frac{i}{\pi\left(m-n_{0}\right)}\right. \\
& \left.\times \exp \left[-i k\left(m-n_{0}\right) f\left(x^{\prime}, y^{\prime}\right)\right]\right\} \\
& \times \exp \left[-i k\left(p x^{\prime}+q y^{\prime}\right)\right] \mathrm{d} x^{\prime} \mathrm{d} y^{\prime} \\
= & \frac{i \psi_{0} F_{0}}{2 m}\left\{\lambda^{2} \delta(p) \delta(q) \delta\left(m-n_{0}\right)+\frac{i}{\pi\left(m-n_{0}\right)}\right. \\
& \times \int_{-\infty}^{\infty} \int_{-\infty}^{\infty} \exp \left[-i k\left(m-n_{0}\right) f\left(x^{\prime}, y^{\prime}\right)\right] \\
& \left.\times \exp \left[-i k\left(p x^{\prime}+q y^{\prime}\right)\right] \mathrm{d} x^{\prime} \mathrm{d} y^{\prime}\right\} .
\end{aligned}
$$

On substitution from the first term in Eq. (18) into Eq. (9) we get

$$
\begin{aligned}
\psi_{s}(\mathbf{x})= & \frac{-i \lambda^{2} \psi_{0} F_{0}}{2} \times \int_{-\infty}^{\infty} \int_{-\infty}^{\infty} \frac{\delta(p) \delta(q) \delta\left(m-n_{0}\right)}{m} \\
& \times \exp [i k(p x+q y+m z)] \mathrm{d} p \mathrm{~d} q \\
= & -i \lambda \psi_{0} F_{0} \mathrm{~d} \exp (i k z) / 2
\end{aligned}
$$

where we have used the fact that the remaining delta function, $\delta(0)=d / \lambda$, comes from an integral that represents the depth of the sea in wavelengths. Finally, by substitution from relation (13) and Eq.
(19) into Eq. (3) we have an expression for the total field transmitted out of the sea into the air, but neglecting the contribution from the second term in Eq. (18). This gives

$$
\psi_{t}(\mathbf{x})=\left(1-i \lambda F_{0} \mathrm{~d} / 2\right) \psi_{0} \exp (i k z) .
$$

Clearly Eq. (20) gives the specular component of the total transmitted field that is not scattered by the sea waves. Under most actual conditions this contribution will be small. The field that is due to scattering from the sea waves is given by substitution from the second term in Eq. (18) into Eq. (9) to yield

$$
\begin{aligned}
\psi_{s}(\mathbf{x})= & \frac{\psi_{0} F_{0}}{2 \pi} \int_{-\infty}^{\infty} \int_{-\infty}^{\infty}\left\{\frac{1}{m\left(m-n_{0}\right)}\right. \\
& \left.\times \int_{-\infty}^{\infty} \int_{-\infty}^{\infty} \exp \left[-i k\left(m-n_{0}\right) f\left(x^{\prime}, y^{\prime}\right)\right] \mathrm{d} x^{\prime} \mathrm{d} y^{\prime}\right\} \\
& \times \exp [i k(p x+q y+m z)] \mathrm{d} p \mathrm{~d} q .
\end{aligned}
$$

The integral over $p$ and $q$ in Eq. (21) is just the summation over the plane waves of light that have been scattered in different directions by the sea waves. The factors of the kernel in braces give the angular spectrum of these plane waves. Thus the angular spectrum of the scattered light is proportional to the integral over $x^{\prime}$ and $y^{\prime}$ in Eq. (21).

This integral over $x^{\prime}$ and $y^{\prime}$ can be evaluated asymptotically for large $k$ by the stationary phase method. Each stationary point in the phase of the kernel contributes to the amplitude of a single plane wave that propagates in the direction given by the unit vector p. To find the stationary points, it is necessary to set both of the first derivatives of the phase with respect to $x^{\prime}$ and $y^{\prime}$ equal to zero and then solve the two resulting equations simultaneously to get the stationary points for $x^{\prime}$ and $y^{\prime}$, i.e.,

$$
\begin{aligned}
& \frac{\partial f\left(x^{\prime}, y^{\prime}\right)}{\partial x^{\prime}}=\frac{-p}{m-n_{0}}, \\
& \frac{\partial f\left(x^{\prime}, y^{\prime}\right)}{\partial y^{\prime}}=\frac{-q}{m-n_{0}} .
\end{aligned}
$$

Equations (22) give the location of the points on the $z=0$ plane that have the proper wave slope to scatter the incident plane wave in the direction given by the unit vector $\mathbf{p}=(p, q, m)$. It can also be interpreted as an equation that gives the directions of the scattered plane wave, $\mathbf{p}=(p, q, m)$, for a single incident plane wave that propagates in the direction $p_{1}=$ $(0,0,1)$ as a function of the local slope at point $\left(x^{\prime}, y^{\prime}\right)$ as given by the sea waveform $f\left(x^{\prime}, y^{\prime}\right)$. Interestingly, we can show that Eqs. (22) turn out to be formally identical to Snell's law at each point $\left(x^{\prime}, y^{\prime}\right)$.

To show this we need only identify the unit vectors that are normal to incident and scattered plane waves with unit vectors in the direction of incident and refracted light rays at some point $\left(x^{\prime}, y^{\prime}\right)$, respectively. Plane-wave normals and light rays are related by the 
fact that the field amplitude at any point in the far field of the origin is proportional to the complex amplitude of the plane wave that propagates in the direction of the point from the origin, as shown by Eq. (44) in Ref. 12. Thus we can associate plane-wave normals near the origin with light rays from the origin to points in the direction of these far-field points. The formal identity of Eq. (22) with Snell's law can then be shown by considering the scalar components of the vector form of Snell's law, ${ }^{13}$ i.e.,

$$
\mathbf{n} \times \mathbf{p}=n_{0}\left(\mathbf{n} \times \mathbf{p}_{i}\right),
$$

where the $x$ and $y$ components of the unit vector $\mathbf{n}$, normal to the surface of the water, are given by

$$
\begin{aligned}
& \frac{\partial f\left(x^{\prime}, y^{\prime}\right)}{\partial x^{\prime}}=-n_{x}, \\
& \frac{\partial f\left(x^{\prime}, y^{\prime}\right)}{\partial y^{\prime}}=-n_{y},
\end{aligned}
$$

$\mathbf{p}_{i}$ is the unit vector in the direction of the incident light ray, and $\mathbf{p}$ is the unit vector in the direction of the refracted light ray as shown in Fig. 3. Since we can associate the unit vectors in the directions of these light rays with the normals to the incident and scattered plane waves, we can set $\mathbf{p}_{i}=(0,0,1)$ as assumed in relation (13). This procedure leads to three scalar equations. Two are identical to those in Eq. (22) and the third can be derived from the first two.

We have shown that scattering theory can properly account for both the specular and scattered parts of the transmitted laser beam. Moreover, we have shown that the refraction of the field at the air-sea boundary, as given by Snell's law, is treated by Eq. (21) for the scattered field. Refraction does not follow from scattering theory if the Born approximation is used, but it clearly does so if the modified Born approximation assumed here is used instead. Finally, Eq. (21) also accounts for the effects of wave theory diffraction of the incident light from the sea waves. To show this requires further evaluation of this equation. However, without knowing $f(x, y)$, we can-

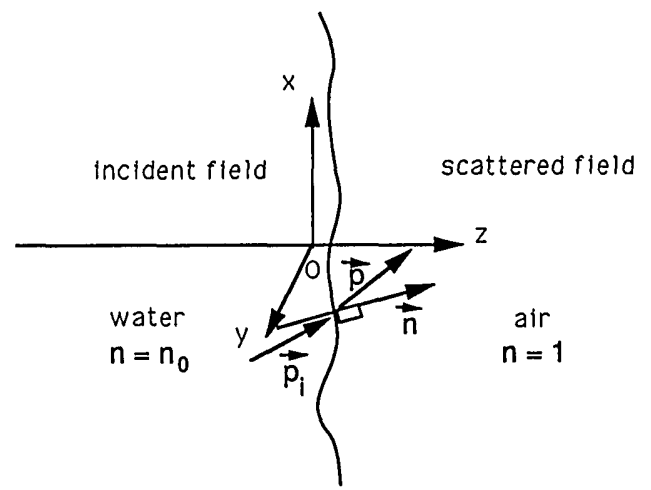

Fig. 3. Unit vectors appearing in Eq. (23) for the vector Snell's law. not integrate Eq. (21) to calculate the radiant intensity of the scattered field.

We should remember that the sea moves in time. To calculate the radiant intensity of the light scattered from the waves at each instant of time it would be necessary to know $f(x, y)$, the precise two-dimensional waveform for the sea surface at that moment. This is almost never known although considerable data that describe properties of this function such as the squared modulus of the averaged two-dimensional Fourier spectrum of $f(x, y)$ have been measured and recorded. ${ }^{14}$ Fortunately, the instantaneous radiant intensity is not usually of primary interest. Far more important is the time-averaged radiant intensity of the time-fluctuating light field. For this it is convenient to employ the quasihomogeneous source model to describe the statistical behavior of the sea waves and then employ the methods of the theory of partial coherence to deal with the partially coherent scattered light. This is described in Section 4. From this calculation we see that this scattering theory gives diffraction effects as well as refraction.

\section{Scattering of a Single Plane Wave from Quasi-Homogeneous Random Sea Waves}

If we assume that it is only the time-averaged radiant intensity of the light scattered from the sea waves that we can measure, and if we assume that the period of this time average is long relative to the fluctuations of the light that is due to the motion of the sea waves, then the theory of partial coherence provides powerful methods for predicting the measured data.

We assume that the sea waves move randomly in time so that the scattering potential in Eq. (12) can be treated as a random process. Furthermore, we assume that the process is ergodic so that ensemble averages can be used in place of time averages. Finally we assume that the sea waves move slowly relative to the period of the monochromatic incident field and fast relative to the averaging time of the light detectors that are used to measure the radiant intensity in the tar field. ${ }^{15}$ Under these conditions it is useful ${ }^{16}$ to describe the scattering potential by use of the cross-spectral density function

$$
W_{F}\left(\mathbf{x}_{1}{ }^{\prime}, \mathbf{x}_{2}{ }^{\prime}\right)=\left\langle F\left(\mathbf{x}_{1}{ }^{\prime}\right) F^{*}\left(\mathbf{x}_{2}{ }^{\prime}\right)\right\rangle,
$$

as is usually done for fluctuating fields, where the angle brackets denote an ensemble average. The light from the coherent laser beam, after scattering from the sea waves, becomes partially coherent. Thus it is useful to define, in the usual manner [viz., Ref. 10, Eq. (76)], the cross-spectral density function

$$
W_{s}\left(\mathbf{x}_{1}{ }^{\prime}, \mathbf{x}_{2}{ }^{\prime}\right)=\left\langle\psi_{s}\left(\mathbf{x}_{1}{ }^{\prime}\right) \psi_{s}{ }^{*}\left(\mathbf{x}_{2}{ }^{\prime}\right)\right\rangle,
$$

and the angular correlation function, ${ }^{17}$

$$
\mathscr{A}\left(\mathbf{p}_{1}, \mathbf{p}_{2}\right)=\left\langle A\left(\mathbf{p}_{1}\right) A^{*}\left(\mathbf{p}_{2}\right)\right\rangle
$$

for the scattered field. On substitution from Eqs. 
(10) and (12) into Eq. (27) we get

$$
\begin{aligned}
\mathscr{A}\left(\mathbf{p}_{1}, \mathbf{p}_{2}\right) & \\
= & \frac{1}{\lambda^{2} m_{1} m_{2}} \int_{-\infty}^{\infty} \int_{-\infty}^{\infty} \int_{-\mathrm{d}}^{z+} \int_{-\infty}^{\infty} \int_{-\infty}^{\infty} \int_{-\mathrm{d}}^{z+} W_{F}\left(\mathbf{x}_{1}{ }^{\prime}, \mathbf{x}_{2}{ }^{\prime}\right) \\
& \times \bar{\psi}_{i}\left(\mathbf{x}_{1}{ }^{\prime}\right) \bar{\psi}_{i}{ }^{*}\left(\mathbf{x}_{2}{ }^{\prime}\right) \\
& \times \exp \left[-i k\left(\mathbf{p}_{1} \cdot \mathbf{x}_{1}{ }^{\prime}-\mathbf{p}_{2}{ }^{*} \cdot \mathbf{x}_{2}{ }^{\prime}\right)\right] \mathrm{d}^{3} \mathbf{x}_{1}{ }^{\prime} \mathrm{d}^{3} \mathbf{x}_{2}{ }^{\prime} .
\end{aligned}
$$

We now assume that the modified incident light field is a plane wave that propagates in a direction given by the unit vector $\mathbf{p}_{i}=\left(p_{i}, q_{i}, m_{i}\right)$, i.e.,

$$
\psi(\mathbf{x})=\psi_{i} \exp \left(i k n_{0} \mathbf{p}_{i} \cdot \mathbf{x}\right) .
$$

On substitution from Eq. (29) into Eq. (28), we have

$$
\begin{aligned}
\mathscr{A}\left(\mathbf{p}_{1}, \mathbf{p}_{2}\right) & \\
= & \frac{\left|\psi_{i}\right|^{2}}{\lambda^{2} m_{1} m_{2}{ }^{*}} \int_{-\infty}^{\infty} \int_{-\infty}^{\infty} \int_{-\mathrm{d}}^{z+} \int_{-\infty}^{\infty} \int_{-\infty}^{\infty} \int_{-\mathrm{d}}^{z+} W_{F}\left(\mathbf{x}_{1}{ }^{\prime}, \mathbf{x}_{2}{ }^{\prime}\right) \\
& \times \exp \left[-i k\left[\left(\mathbf{p}_{1}-n_{0} \mathbf{p}_{i}\right) \cdot \mathbf{x}_{1}{ }^{\prime}\right.\right. \\
& \left.\left.-\left(\mathbf{p}_{2}{ }^{*}-n_{0} \mathbf{p}_{i}\right) \cdot \mathbf{x}_{2}{ }^{\prime}\right)\right] \mathrm{d}^{3} \mathbf{x}_{1}{ }^{\prime} \mathrm{d}^{3} \mathbf{x}_{2}{ }^{\prime} \\
= & \frac{\left|\psi_{i}\right|^{2}}{\lambda^{4} m_{1} m_{2}{ }^{*}} \int_{-\infty}^{\infty} \int_{-\infty}^{\infty} \int_{-\infty}^{\infty} \int_{-\infty}^{\infty} \mathscr{G}\left(x_{1}{ }^{\prime}, y_{1}{ }^{\prime}, x_{2}{ }^{\prime}, y_{2}{ }^{\prime}\right) \\
& \times \exp \left\{-i k\left[\left(p_{1}-n_{0} p_{i}\right) x_{1}{ }^{\prime}+\left(q_{1}-n_{0} q_{i}\right) y_{1}{ }^{\prime}\right.\right. \\
& \left.\left.-\left(p_{2}-n_{0} p_{i}\right) x_{2}{ }^{\prime}-\left(q_{2}-n_{0} q_{i}\right) y_{2}{ }^{\prime}\right]\right] \mathrm{d} x_{1}{ }^{\prime} \mathrm{d} y_{1}{ }^{\prime} \mathrm{d} x_{2}{ }^{\prime} \mathrm{d} y_{2}{ }^{\prime},
\end{aligned}
$$

where the correlation function for the surface scattering function is defined by

$$
\begin{aligned}
& \mathscr{G}\left(x_{1}{ }^{\prime}, y_{1}{ }^{\prime}, x_{2}{ }^{\prime}, y_{2}{ }^{\prime}\right) \\
&=\lambda^{2} \int_{-\mathrm{d}}^{z+} \int_{-\mathrm{d}}^{z+} W_{F}\left(\mathbf{x}_{1}{ }^{\prime}, \mathbf{x}_{2}{ }^{\prime}\right) \exp \left\{-i k\left[\left(m_{1}-n_{0} m_{i}\right) z_{1}{ }^{\prime}\right.\right. \\
&\left.\left.\quad-\left(m_{2}{ }^{*}-n_{0} m_{i}\right) z_{2}{ }^{\prime}\right]\right\} \mathrm{d} z_{1}{ }^{\prime} \mathrm{d} z_{2}{ }^{\prime} .
\end{aligned}
$$

This function is a correlation function for the sea waves between two points $\left(x_{1}{ }^{\prime}, y_{1}{ }^{\prime}\right)$ and $\left(x_{2}{ }^{\prime}, y_{2}{ }^{\prime}\right)$ on the sea surface. The dependence on $m_{1}$ and $m_{2}$ would be expected cause this junction to drop off sharply as either of these components differed appreciably from $n_{0} m_{i}$ in the same manner as given by Eq. (17) for a normally incident plane wave. We assume that this dependence on $m_{1}$ and $m_{2}$ will not be significant in the following analysis just as it was not in the calculation leading to Eqs. (20) and (21). For simplicity, the dependence on $m_{1}$ and $m_{2}$ will be dropped from the notation. Rather than trying to evaluate Eq. (31) in a manner similar to Eq. (15) in the Section 3, we now simply assume that $\mathscr{G}$ is quasi-homogeneous over its dependence on $\left(x_{1}{ }^{\prime}, y_{1}{ }^{\prime}\right)$ and $\left(x_{2}{ }^{\prime}, y_{2}{ }^{\prime}\right)$, i.e.,

$$
\mathscr{G}\left(x_{1}{ }^{\prime}, y_{1}{ }^{\prime}, x_{2}{ }^{\prime}, y_{2}{ }^{\prime}\right)=I_{G}\left(x_{+}, y_{+}\right) \mu_{G}\left(x_{-}, y_{-}\right),
$$

where the variables on the two sides of this equation are related by

$$
\begin{array}{ll}
x_{+}=\frac{\left(x_{1}{ }^{\prime}+x_{2}^{\prime}\right)}{2}, & y_{+}=\frac{\left(y_{1}^{\prime}+y_{2}^{\prime}\right)}{2}, \\
x_{-}=\left(x_{1}^{\prime}-x_{2}^{\prime}\right), & y_{-}=\left(y_{1}^{\prime}-y_{2}^{\prime}\right), \\
x_{1}^{\prime}=x_{+}+\frac{x_{-}}{2}, & y_{1}^{\prime}=y_{+}+\frac{y_{-}}{2}, \\
x_{2}^{\prime}=x_{+}-\frac{x_{-}}{2}, & y_{2}^{\prime}=y_{+}-\frac{y_{-}}{2} .
\end{array}
$$

It is assumed, as usual, that $\mu_{G}\left(x_{-}, y_{-}\right)$is a fast function ${ }^{18}$ relative to $I_{G}\left(x_{+}, y_{+}\right)$. In analogy to the usual nomenclature for the correlation functions for fluctuating fields in coherence theory, $I_{G}\left(x_{+}, y_{+}\right)$is called the intensity of the sea waves and $\mu_{G}\left(x_{-}, y_{-}\right)$, which is a properly normalized correlation coefficient for the fluctuations of the sea waves, is called the complex degree of spectral coherence for these waves. On substitution from Eq. (32) into Eq. (30) and transforming to the plus and minus coordinates by using Eq. (33), we find, using the well-known Fouriertransform properties of a quasi-homogeneous source function, ${ }^{3}$ that

$$
\mathscr{A}\left(\mathbf{p}_{1}, \mathbf{p}_{2}\right)=\frac{\left|\psi_{i}\right|^{2}}{m_{1} m_{2}} \tilde{I}_{G}\left(\frac{p_{-}}{\lambda}, \frac{q_{-}}{\lambda}\right) \times \tilde{\mu}_{G}\left(\frac{p_{+}}{\lambda}, \frac{q_{+}}{\lambda}\right),
$$

where the coordinates on two sides of this equation are related by

$$
\begin{array}{ll}
p_{-}=p_{1}-p_{2}, & q_{-}=q_{1}-q_{2}, \\
p_{+}=\frac{\left(p_{1}+p_{2}-2 n_{0} p_{i}\right)}{2}, & q_{+}=\frac{\left(q_{1}+q_{2}-2 n_{0} q_{i}\right)}{2}, \\
p_{1}=p_{+}+n_{0} p_{i}+\frac{p_{-}}{2}, & q_{1}=q_{+}+n_{0} q_{i}+\frac{q_{-}}{2}, \\
p_{2}=p_{+}+n_{0} p_{i}-\frac{p_{-}}{2}, & q_{2}=q_{+}+n_{0} q_{i}-\frac{q_{-}}{2},
\end{array}
$$

and the tilde indicates a two-dimensional Fourier transform, such as

$$
\begin{aligned}
\tilde{I}_{G}\left(\frac{p_{-}}{\lambda}, \frac{q_{-}}{\lambda}\right)= & \frac{1}{\lambda^{2}} \int_{-\infty}^{\infty} \int_{-\infty}^{\infty} I_{G}\left(x_{+}, y_{+}\right) \\
& \times \exp \left[-i k\left(p_{-} x_{+}+q_{-} y_{+}\right)\right] \mathrm{d} x_{+} \mathrm{d} y_{+}, \\
\tilde{\mu}_{G}\left(\frac{p_{+}}{\lambda}, \frac{q_{+}}{\lambda}\right)= & \frac{1}{\lambda^{2}} \int_{-\infty}^{\infty} \int_{-\infty}^{\infty} \mu_{G}\left(x_{-}, y_{-}\right) \\
& \times \exp \left[-i k\left(p_{+} x_{-}+q_{+} y_{-}\right)\right] \mathrm{d} x_{-} \mathrm{d} y_{-} .
\end{aligned}
$$

The radiant intensity in a direction given by the unit vector $\mathbf{s}=\left(s_{x}, s_{y}, s_{z}\right)$ from the origin as shown in Fig. 4 is given as a function of the angular correlation 


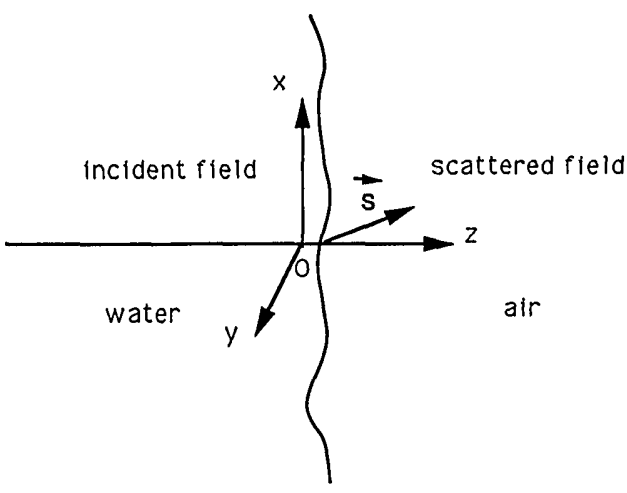

Fig. 4. Unit vector from the origin in the direction of the radiant intensity carried by the scattered field into its radiation zone in Eq. (39).

function by [see Ref. 12, Eq. (44)]

$$
J_{\omega}(\mathbf{s})=\lambda^{2} s_{z}^{2} \mathscr{A}(\mathbf{s}, \mathbf{s}) ;
$$

therefore, on substitution from Eq. (34) into Eq. (38) we have our result:

$$
J_{\omega}(\mathbf{s})=\lambda^{2}\left|\psi_{i}\right|^{2} \tilde{I}_{G}(0,0) \tilde{\mu}_{G}\left[\frac{\left(s_{x}-n_{0} p_{i}\right)}{\lambda}, \frac{\left(s_{y}-n_{0} q_{i}\right)}{\lambda}\right] .
$$

From Eq. (39) it is clear that the angular dependence of the radiant intensity of the scattered laser light in the far field is determined completely by the Fourier transform of the complex degree of spectral coherence for the sea wave fluctuations. All other factors in Eq. (39) are constants of direction. The physical significance of Eq. (39) is illustrated schematically in Fig. 5.

The index of refraction in Eq. (39) accounts for refraction of the incident plane wave at the interface as described in Section 3. However, Eq. (39) also accounts for wave theory diffraction effects on the scattered light from the interaction with sea waves. These effects are not treated by the usual geometrical optics models. The treatment of diffraction effects

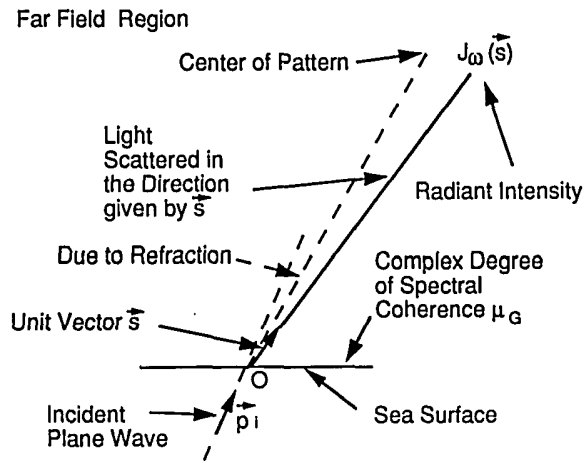

Fig. 5. Schematic diagram of the physical significance of Eq. (39). The radiant intensity as a function of $\mathbf{s}$ for the incident plane wave after scattering from the sea surface is effected only by the Fourier transform of the complex degree of spectral coherence of the sea wave fluctuations. by using Eq. (39) is most easily shown by the following observations. The peak of the scattered field is generally in the same direction as the incident plane wave after refraction at the surface. The field falls off, away from this direction as the Fourier transform of the complex degree of spectral coherence of the sea wave fluctuations. Consider the uncertainty principle for Fourier conjugate variables, i.e.,

$$
\Delta s \Delta x \geq \lambda /(2 \pi),
$$

where $\Delta s$ is the variance of a typical component of unit vector $\mathbf{s}$ in Eq. (39), and $\Delta x$ is the variance of the Fourier conjugate [see Eq. (37)] component of $\mathbf{x}_{-}$, the separation of the correlated points on the surface of the sea waves. If the sea waves are correlated over dimensions of the order of some radius $\delta$, which is large relative to a wavelength of the incident coherent light, then from inequality (40) it is clear that $\Delta s$ will typically be small so that the scattering will be mostly in the direction $\mathbf{s}=n_{0} \mathbf{p}_{i}$ given by geometrical refraction of the incident plane wave. However, as $\delta$ becomes smaller, it is clear from inequality (40) that $\Delta s$ must increase. Thus, from inequality $(40)$ we see that the divergence angle for the scattered light beam $\theta$ must become larger in such a manner that $\theta$ satisfies the well-known equation for the diffraction angle of coherent light diffracted from an aperture with a diameter $\delta$, i.e.,

$$
\sin \theta \geq \lambda /(2 \pi \delta) .
$$

Clearly, therefore, Eq. (39) accounts for diffraction effects that are due to the interaction of the light wave with the sea wave, which are not accounted for by geometrical optics. It appears that the correlation length $\delta$ for the wave motion behaves like the diameter of a diffracting aperture with respect to the spread of a scattered laser beam.

\section{Scattering of a Laser Beam from Quasi-Homogeneous Sea Waves}

The calculation presented in Section 4 can be generalized to treat a coherent beam rather than just a single plane wave. Any linearly polarized electromagnetic beam field can be expanded into an angular spectrum of plane waves in the form ${ }^{19,20}$

$$
\begin{aligned}
\bar{\psi}_{i}(\mathbf{x})= & \int_{-\infty}^{\infty} \int_{-\infty}^{\infty} A_{i}\left(p_{i}, q_{i}\right) \\
& \times \exp \left[i k n_{0}\left(p_{i} x+q_{i} y+m_{i} z\right)\right] \mathrm{d} p_{i} \mathrm{~d} q_{i} .
\end{aligned}
$$

We use the field in Eq. (42) to represent the modified incident beam field inside the sea. The expression for the angular spectrum for most common beams is well known. For example, the angular spectrum for a simple Gaussian beam that propagates with a beam axis coincident with the $z$ axis and is focused in the $z=0$ plane is ${ }^{19}$

$$
A_{i}\left(p_{i}, q_{i}\right)=A_{0} \exp \left[-k^{2} n_{0}^{2} \sigma^{2}\left(p_{i}{ }^{2}+q_{i}{ }^{2}\right) / 2\right],
$$


where $A_{0}$ is a constant and $\sigma$ is the radius of the beam in its focal plane. We again assume that the sea waves are quasi-homogeneous so that Eq. (32) is valid. Thus, on substitution from Eq. (43) into Eq. (28) while Eqs. (31) and (32) are used, we have

$\mathscr{A}\left(\mathbf{p}_{1}, \mathbf{p}_{2}\right)$

$$
\begin{aligned}
= & \frac{1}{\lambda^{2} m_{1} m_{2}} \int_{-\infty}^{\infty} \int_{-\infty}^{\infty} A_{i}\left(p_{i}, q_{i}\right) \int_{-\infty}^{\infty} \int_{-\infty}^{\infty} A_{i}{ }^{*}\left(p_{i}{ }^{\prime}, q_{i}{ }^{\prime}\right) \\
& \times \int_{-\infty}^{\infty} \int_{-\infty}^{\infty} \int_{d}^{z+} \int_{-\infty}^{\infty} \int_{-\infty}^{\infty} \int_{d}^{z+} W_{F}\left(\mathbf{x}_{1}{ }^{\prime}, \mathbf{x}_{2}{ }^{\prime}\right) \\
& \times \exp \left[-i k\left[\left(\mathbf{p}_{1}-n_{0} \mathbf{p}_{i}\right) \cdot \mathbf{x}_{1}{ }^{\prime}-\left(\mathbf{p}_{2}{ }^{*}-n_{0} \mathbf{p}_{i}{ }^{\prime}\right) \cdot \mathbf{x}_{2}{ }^{\prime}\right)\right] \\
& \times d^{3} \mathbf{x}_{1}{ }^{\prime} d^{3} \mathbf{x}_{2}{ }^{\prime} d^{2} \mathbf{p}_{i} d^{2} \mathbf{p}_{i}{ }^{\prime} \\
= & \frac{1}{\lambda^{4} m_{1} m_{2}} \int_{-\infty}^{\infty} \int_{-\infty}^{\infty} A_{i}\left(p_{i}, q_{i}\right) \int_{-\infty}^{\infty} \int_{-\infty}^{\infty} A_{i}{ }^{*}\left(p_{i}{ }^{\prime}, q_{i}{ }^{\prime}\right) \\
& \times \int_{-\infty}^{\infty} \int_{-\infty}^{\infty} \int_{-\infty}^{\infty} \int_{-\infty}^{\infty} \mathscr{G}\left(x_{1}{ }^{\prime}, y_{1}{ }^{\prime}, x_{2}{ }^{\prime}, y_{2}{ }^{\prime}\right) \\
& \times \exp \left\{-i k\left[\left(p_{1}-n_{0} p_{i}\right) x_{1}{ }^{\prime}+\left(q_{1}-n_{0} q_{i}\right) y_{1}{ }^{\prime}\right.\right. \\
& \left.\left.-\left(p_{2}-n_{0} p_{i}{ }^{\prime}\right) x_{2}{ }^{\prime}+\left(q_{2}-n_{0} q_{i}{ }^{\prime}\right) y^{\prime}\right]\right\} \\
& \times \mathrm{d} x_{1}{ }^{\prime} \mathrm{d} y_{1}{ }^{\prime} \mathrm{d} x_{2}{ }^{\prime} \mathrm{d} y_{2}{ }^{\prime} \mathrm{d}^{2} \mathbf{p}_{i} \mathrm{~d}^{2} \mathbf{p}_{i}{ }^{\prime} \\
= & \frac{1}{m_{1} m_{2}} \int_{-\infty}^{\infty} \int_{-\infty}^{\infty} A_{i}\left(p_{i}, q_{i}\right) \int_{-\infty}^{\infty} \int_{-\infty}^{\infty} A_{i}{ }^{*}\left(p_{i}{ }^{\prime}, q_{i}{ }^{\prime}\right) \\
& \times \tilde{\mu}_{G}\left(\frac{p_{+}-n_{0} p_{i+}}{\lambda}, \frac{q_{+}-n_{0} q_{i+}}{\lambda}\right) \\
& \times \tilde{I}_{G}\left(\frac{p_{-}-n_{0} p_{i-}}{\lambda}, \frac{q_{-}-n_{0} q_{i-}}{\lambda}\right) \mathrm{d}^{2} \mathbf{p}_{i} \mathrm{~d}^{2} \mathbf{p}_{i}{ }^{\prime}, \\
&
\end{aligned}
$$

where the tilde again denotes a Fourier transform as defined in Eqs. (36) and (37). If we assume that the function $I_{G}\left(x_{+}, y_{+}\right)$in Eq. (32) is so slowly varying that it can be treated as a constant over the illuminated area on the sea surface, then its Fourier transform is given by

$$
\tilde{I}_{G}\left(\frac{p_{-}-n_{0} p_{i-}}{\lambda}, \frac{q_{-}-n_{0} q_{i_{-}}}{\lambda}\right)=\delta^{2}\left(\mathbf{p}_{-}-n_{0} \mathbf{p}_{i-}\right),
$$

where we use the two-dimensional vectors $\mathbf{p}_{i+}$ and $\mathbf{p}_{i-}$ with components given by

$$
\begin{aligned}
p_{i-}=p_{i}-p_{i}{ }^{\prime}, & q_{i-}=q_{i}-q_{i}{ }^{\prime}, \\
p_{i+}=\frac{\left(p_{i}+p_{i}{ }^{\prime}\right)}{2}, & q_{i+}=\frac{\left(q_{i}+q_{i}{ }^{\prime}\right)}{2}, \\
p_{i}=p_{i+}+\frac{p_{i-}}{2}, & q_{i}=q_{i+}+\frac{q_{i-}}{2}, \\
p_{i}{ }^{\prime}=p_{i+}-\frac{p_{i-}}{2}, & q_{i}{ }^{\prime}=q_{i+}-\frac{q_{i-}}{2},
\end{aligned}
$$

and the two-dimensional vectors $\mathbf{p}_{+}$and $\mathbf{p}_{-}$with components given by

$$
\begin{array}{ll}
p_{-}=p_{1}-p_{2}, & q_{-}=q_{1}-q_{2}, \\
p_{+}=\frac{\left(p_{1}+p_{2}\right)}{2}, & q_{+}=\frac{\left(q_{1}+q_{2}\right)}{2}, \\
p_{1}=p_{+}+\frac{p_{-}}{2}, & q_{1}=q_{+}+\frac{q_{-}}{2}, \\
p_{2}=p_{+}-\frac{p_{-}}{2}, & q_{2}=q_{+}-\frac{q_{-}}{2} .
\end{array}
$$

By transforming coordinates in Eq. (44), using Eqs. (46) and (47), and then using Eq. (45), we can evaluate the integrals in Eq. (44) to get

$$
\begin{aligned}
\mathscr{A}\left(\mathbf{p}_{1}, \mathbf{p}_{2}\right)= & \frac{1}{n_{0}^{2} m_{1} m_{2}} \int_{-\infty}^{\infty} \int_{-\infty}^{\infty} \tilde{\mu}_{G}\left(\frac{\mathbf{p}_{+}-n_{0} \mathbf{p}_{i+}}{\lambda}\right) \\
& \times A_{i}\left(\mathbf{p}_{i+}+\frac{\mathbf{p}_{-}}{2 n_{0}}\right) A_{i}^{*}\left(\mathbf{p}_{i+}-\frac{\mathbf{p}_{-}}{2 n_{0}}\right) \mathrm{d}^{2} \mathbf{p}_{i+} .
\end{aligned}
$$

Finally, by substitution from Eq. (48) into Eq. (38), we have the result

$$
J_{\omega}(\mathbf{s})=\frac{\lambda^{2}}{n_{0}{ }^{4}} \tilde{\mu}_{G}(\mathbf{s} / \lambda) *\left|A_{i}\left(\mathbf{s} / n_{0}\right)\right|^{2},
$$

where the asterisk denotes a convolution of the form

$$
\begin{aligned}
\tilde{\mu}_{G}(\xi) *\left|A_{i}(\xi)\right|^{2} & \\
& =\int_{-\infty}^{\infty} \int_{-\infty}^{\infty} \tilde{\mu}_{G}\left(\xi-\xi^{\prime}\right) A_{i}\left(\xi^{\prime}\right) A_{i}^{*}\left(\xi^{\prime}\right) \mathrm{d}^{2} \xi^{\prime} .
\end{aligned}
$$

From Eq. (49) we note that the radiant intensity of the scattered light in the direction $\mathbf{s}$ from the origin is proportional to the Fourier transform of the complex degree of spectral coherence for the sea waves convolved with the squared magnitude of the angular spectrum of the incident field. The transmitted beam spread is increased due to refraction at the interface as shown by the rescaling of incident angular spectrum by the index of refraction of water. This beam spread is also broadened by diffraction scattering from the sea waves as shown by the convolution of the angular spectrum with the Fourier transform of the complex degree of spectral coherence for sea waves. By carrying out the convolution for the simple case of a single nonvanishing plane wave, Eq. (49) becomes identical to Eq. (39).

It is important to note that, if the Fourier transform of the complex degree of spectral coherence in Eq. (49) is broader than the rescaled angular spectrum for the incident light beam, then it is this function that determines the width of the radiant intensity as a function of direction. Thus the diffraction of the beam from the surface waves determines the spread of the scattered beam as it propagates away from the sea surface and not simple refraction 
at the water-air interface as is usually assumed when we deal with sea scatter problems. This should occur for quasi-homogeneous sea wave statistics if the correlation length for the fluctuations of the sea waves is not long relative to the wavelength of radiation. For light scattering from the usual ocean waves this might not usually be the case, but it might be for much longer wavelength radiation or in the presence of extreme sea wave turbulence.

\section{Conclusions}

The scattering of coherent light from sea waves has been treated by using the scattering theory in an approximation that is a significant improvement over the first Born approximation. In Section 3 we have seen that this theory gives a result that is similar to the usual geometrical optics refraction result if the sea wave function is assumed to be deterministic. This same calculation also gives the specular component of the incident light that is transmitted by the wave surface and the diffraction of the light waves that is due to the effect of scattering from the sea waves.

The effects of diffraction that are due to the scattering of light from sea waves are best seen in the statistical solution to the scattering problem that was developed in Sections 4 and 5. Mathematically simple results were found for the radiant intensity for both a single plane wave in Section 4 and for an arbitrary beam field composed of a complete angular spectrum of plane waves in Section 5. It was found that, in addition to the effects of refraction that are usually considered in the familiar theories based on geometrical optics models, effects on the radiant intensity that are due to diffraction by the sea waves. For quasi-homogeneous sea waves these effects are dependent only on the Fourier transform of the complex degree of spectral coherence for sea wave fluctuations on the sea's surface. The diffraction effects can dominate the spread of the scattered light beam as it propagates away from the sea surface if the correlation length for the sea waves over the surface is of the order of a wavelength of the incident radiation or smaller.

This research was conducted while W. H. Carter was a visiting scientist at the Johns Hopkins University Applied Physics Laboratory.

\section{References}

1. E. Wolf, "Three dimensional structure determination of semitransparent objects from holographic data," Opt. Commun. 1, 153-156 (1969).

2. E. Wolf, "Coherence and radiometry," J. Opt. Soc. Am. 68, 6-17 (1978).

3. W. H. Carter, and E. Wolf, "Coherence and radiometry with quasihomogeneous planar sources," J. Opt. Soc. Am. 67, 785-796 (1977).

4. S. Karp, "Optical communications between underwater and above water surface (satellite) terminals," IEEE Trans Commun. COM-24, 66-81 (1976).

5. Y. Guern, J. Lotrain, A. Bideau-Mehu, and J. Cariou, "Irradiance of a laser beam through a wavy ocean surface," Appl. Opt. 24, 655-659 (1985).

6. C. Cox and W. Munk, "Measurement of the roughness of the sea surface from photographs of the Sun's glitter," J. Opt. Soc. Am. 44, 838-854 (1954).

7. J. Wu, "Sea surface slope and equilibrium wind wave spectra," Phys. Fluids 15, 75-80 (1971).

8. J. D. Jackson, Classical Electrodynamics (Wiley, New York, 1962), Eq. (9.3).

9. H. Weyl, "Radiation of electric waves over a plane conductor," Ann. Phys. (Paris) 60, 481-500 (1919).

10. W. H. Carter, "Coherence theory," in The Optical Society of America Handbook of Optics, M. Bass, ed. (McGraw-Hill, New York, 1993).

11. R. N. Bracewell, The Fourier Transform and Its Applications (McGraw-Hill, New York, 1986).

12. E. W. Marchand, and E. Wolf, "Radiometry with sources of any state of coherence," J. Opt. Soc. Am. 64, 1219-1226 (1974).

13. W. T. Welford, Aberrations of the Symmetrical Optical System (Academic, New York, 1974).

14. A. W. Bjerkaas, and F. W. Riedel, "Proposed model for the elevation spectrum of a wind-roughened sea surface," Johns Hopkins University Applied Physics Laboratory Rep. No. STD-R-041 (Applied Physics Laboratory, Johns Hopkins University, Laurel, Md., April 1979).

15. W. H. Carter, "Scattering from quasi-homogenous time fluctuating random media," Opt. Commun. 77, 121-125 (1990).

16. W. H. Carter, and E. Wolf, "Scattering from quasi-homogeneous media," Opt. Commun. 67, 85-90 (1988).

17. E. W. Marchand and E. Wolf, "Angular correlation and the far-zone behavior of partially coherent fields," J. Opt. Soc. Am. 62, 379-385 (1972).

18. E. Wolf and W. H. Carter, "Fields generated by homogeneous and quasi-homogeneous planar secondary sources," Opt. Commun. 50, 131-136 (1984).

19. W. H. Carter, "The electromagnetic field of a gaussian beam with an elliptical cross section," J. Opt. Soc. Am. 62, 11951201 (1972).

20. W. H. Carter, "Electromagnetic beam fields," Opt. Acta 21, 871-892 (1974). 